\title{
The Facial Expression Effect of an Animated Agent on the Decisions Taken in the Negotiation Game
}

\author{
Masahide Yuasa \\ Tokyo Denki University \\ 2-1200 Muzai Gakuendai \\ Inzai, Chiba, JAPAN \\ yuasa@sie.dendai.ac.jp \\ Naoki Mukawa \\ Tokyo Denki University \\ 2-1200 Muzai Gakuendai \\ Inzai, Chiba, JAPAN \\ mukawa@sie.dendai.ac.jp
}

\begin{abstract}
This paper investigates the manner in which decisionmaking is influenced by the impressions given by lifelike agents in negotiation situations. These impressions comprise an agent's facial expressions such as happy and sad, and the history of relationship with the agent. In this paper, we introduce a negotiation game as one of the basic interactions based on the soft game theory. The experimental results reveal that expressions and history significantly influence the receiver's impressions and decision-making. The findings of this study can be beneficial for designing the nonverbal expressions of an animated agent who can negotiate and make a deal with users.
\end{abstract}

\section{Keywords}

Life-like character, animated agent, game theory, facial expression, appearance, negotiation

\section{ACM Classification Keywords}

H5.1. Information interfaces and presentation (e.g., HCI): Multimedia Information Systems. 


\section{Introduction}

Recent years have witnessed the development of animated agents (occasionally known as life-like characters, avatars, interface agents, Embodied Conversational Agents (ECAs), etc.) $[12,13,14]$. These animated agents have been used in a wide range of application areas, including entertainment, e-commerce, and online negotiations. Several researchers have developed models that automatically represent the emotions and facial expressions of animated agents and robots $[9,11,16]$. However, these models have not been used in practical applications and hence the manner in which they influence human behaviors is not clear. In order to evaluate the effect of emotions and facial expressions, we introduce a negotiation game, a typical game involved in game theory. Some of the research conducted on game theory involved the use of faces $[7,10]$. Eckel et al. focused on the abstract facial icons and photo images in the ultimatum game [2]. On the other hand, Howard proposed soft game theory $[5,6]$, in which two players take decisions by considering the positive or negative impressions given to each other in a situation that is similar to the one in Prisoner's Dilemma. While previous research on game theory focused solely on the face maintained in a game, the soft game theory considered only positive and negative emotions.

Therefore, this study takes into account a variety of facial expressions, such as happy and sad, and the history of agent behavior. In this paper, we introduce a variation in facial expressions and the impressions received from opponent agents. Further, we investigate the impressions and decisions of users who are influenced by facial expressions in an online negotiation game. We presently aim to develop animated agents who can substitute humans in an online negotiation game. Negotiation is one of the basic interactions in which both verbal and nonverbal information is exchanged among humans on a daily basis [15].

Additionally, this paper investigates the manner in which an agent's nonverbal information influences users in the negotiation game based on the soft game theory. We define a new decision relevance model in which impression factors such as friendliness, dominance, and trustworthiness influence decisionmaking, as opposed to only positive or negative emotions, in the soft game theory. We believe that an agent's appearance, history, and expression influence the impression factors. The agent's appearance, history, and nonverbal expression that are best suited to gain cooperation from partners in each case can be decided by agent designers and developers.

\section{Decision Relevance Model}

Using the concept of soft game theory, we propose a new decision relevance model for human behavior. As mentioned previously, this paper focuses on an agent's appearance, history, and expression. Here, appearance implies the facial features of a human being or the opponent's face [4]. According to several researchers, each face of agents or robots conveys different impressions $[1,8]$. History implies whether or not a partner is an acquaintance and whether he or she will influence the subsequent negotiation [18]. Finally, expression implies nonverbal expression, including facial expression, posture, gaze behavior, and prosody $[3,17]$. We believe that appearance, history, and expression help to create impressions about the opponent; these impressions influence players' 
decision-making. We use the following definitions and equations to describe these relationships.

$\begin{array}{ll}\text { Message } & M_{k}=\left\{m_{0}, m_{1}, m_{2}, \ldots\right\} \\ \text { Appearance } & A_{k}=\left\{a_{0}, a_{1}, a_{2}, \ldots\right\} \\ \text { History } & H_{k}=\left\{h_{0}, h_{1}, h_{2}, \ldots\right\} \\ \text { Expression } & E_{k}=\left\{e_{0}, e_{1}, e_{2}, \ldots\right\} \\ \text { Strategy } & S_{k}=\left\{s_{0}, s_{1}, s_{2}, \ldots\right\}=\left\{c_{k}, d_{k}\right\}\end{array}$

(In Prisoner's Dilemma, $S_{k}=\left\{c_{k}, d_{k}\right\}$, i.e., cooperation or defect.)

(1) Impression vector: $\quad A_{p} \times H_{p} \times E_{p} \rightarrow I_{-p}=\left(i_{0}, i_{1}, i_{2}\right.$, $\ldots)^{\mathrm{t}}$

(2) Reasoning function: $M_{p} \times I_{-p} \rightarrow S_{-p}$

(3) Decision function: $\quad M_{-p} \times I_{p} \times S_{-p} \rightarrow S_{p}$

table 1. Prisoner's dilemma. The first and second numbers in each cell represent the benefits of player 1 and player 2 , respectively.

\begin{tabular}{|c|c|c|c|}
\hline \multicolumn{2}{|c|}{} & \multicolumn{2}{c|}{ player 2} \\
\cline { 3 - 4 } \multicolumn{2}{|c|}{} & $\begin{array}{c}\mathrm{c}_{2} \\
\text { Cooperate }\end{array}$ & $\begin{array}{c}\mathrm{d}_{2} \\
\text { Defect }\end{array}$ \\
\hline \multirow{2}{*}{ player 1 } & $\mathrm{c}_{1}$ Cooperate & 3,3 & 1,4 \\
\cline { 2 - 4 } & $\mathrm{d}_{1}$ Defect & 4,1 & 2,2 \\
\hline
\end{tabular}

The variation in parameters is indicated by a subscript $(0,1,2, \ldots)$. The subscript " $k$ " represents player 1 or player 2; "p," a player; and "-p," the other player. In soft game theory, players are allowed to use limited messages (" $\mathrm{M}_{\mathrm{k}}$ ") (for example, "I will select cooperate, so please select cooperate") [5]. Impression (" $I_{k}$ ") is a vector, and " $t$ " denotes a transposed vector. The impression vector is represented by the factors of personality (for example, friendliness, trustworthiness, and dominance). For example, when player 1 gives the impressions of $A_{1}, H_{1}$, and $E_{1}$ to player 2 , he/she receives these impressions and they influence $I_{2}$. Subsequently, he/she takes a decision $\mathrm{S}_{2}$ that is influenced by not only $M_{1}$ but also $I_{2}$. Furthermore, for example, in Prisoner's Dilemma (table 1), when both player 1 and player 2 give good impressions to each other, and if player 1 believes that player 2 will select the strategy $S_{2}=c_{2}$ and player 2 , in turn, believes that player 1 will select the strategy $S_{1}=c_{1}$, then " $C_{1}, c_{2}$ " becomes the final scenario. These inferences are in keeping with the concept of soft game theory in that they describe how the players will achieve cooperation $[5,6]$.

\section{Experiment}

This experiment aims to confirm whether history and expression will influence the impressions and decisions of players. In this experiment, we assume that it is sufficient to only confirm Equations (1) and (2) because the user who selects "cooperate" or "defect" (3) is responsible for inferring his/her partner's selection. We conducted the experiment using "gas stations" in the situation chosen for a typical negotiation game. The players own stations $A$ and $B$, respectively. Each owner continues to sell gas at the rate of 100 yen per liter, and each of them notches up sales of 20 million yen. When both the owners sell gas at the rate of 90 yen per liter, each of them notches up sales of 10 million yen. When one owner sells gas at 90 yen per liter, he/she earns up to 30 million yen. Consequently, the other owner suffers a loss of 10 million yen. The subjects of this study played the game with animated agents through a computer network. We informed the subjects that the animated agents were controlled by the subjects' opponents. However, the animated agents 
were controlled by our program, which randomly generated the facial expressions. The subjects were not allowed to communicate with the agents but could use limited expressions and messages. The following is a description of the procedure used in our experiment:

(1) An agent appears with the message that he/she is meeting with the subject for the first or the second time.

(2) The agent declares his/her promise by changing facial expressions (Figure 1).

(3) The subject is required to evaluate his/her impression of the agent.

(4) The subject is required to decide whether the price of the gas should be 100 yen or 90 yen per liter.

(5) The agent selects his/her decision.

(6) The result (cooperation or defect) is displayed.

The following is the relationship between our model and the experiment

Appearance Several agents were designed by deforming many photos that were obtained from magazines and websites. We selected an agent who appeared to be less dominant and less friendly because we wanted the agent to have minimal influence on the subject's decision-making.

History The subjects were required to answer the agents who met them for the first or the second time after the agent selected "cooperate" or "defect." In this experiment, the subjects believed that the agents who appeared were the same but the players were different in some cases. We believe that after an agent selects "cooperate," he/she will give the impression of being trustworthy and cooperation will occur at high rates.
Expression The agent can express himself or herself by using BOW, AVERT, HAPPY, ANGRY, and COOL (Figure 2). The HAPPY may induce a higher friendliness impression and the ANGRY may induce a lower friendliness impression and a higher dominance impression [17]. The BOW and AVERT may induce a lower dominance impression than any other facial expressions [3].

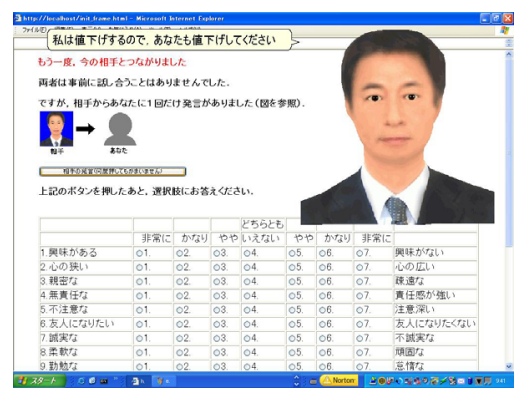

figure 1. The interface of the experiment.

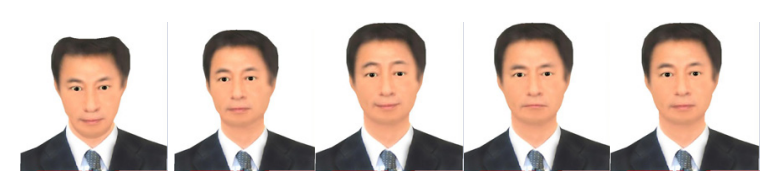

figure 2. Facial expression and gaze direction (BOW, AVERT, HAPPY, ANGRY, and COOL).

\section{Result}

This study involved twelve students from the Department of Computer Science. The experiment involved a total of 456 cases. We analyzed the subjects' impressions. They were required to select the most appropriate response from 20 adjective pairs, using a seven-point semantic differential scale. Factor analysis was used to determine the structure of the data. The 
first dimension assessed the general measurement of trustworthiness; the second dimension, friendliness; and the third dimension, dominance.

We verified the correlation between impression and subjects' selection. The factor score of trustworthiness after an agent selected "cooperate" (AC) was higher than the first time (F) and after he /she selected "defect" (AD). Next, the subjects selected "cooperate" at higher rates in the case of $A C$ (average $=53.9 \%$ ) as compared with $A D(27.4 \%)$ and $F(36.8 \%)$. In particular, the cooperation occurred at the highest rate $(63.1 \%)$ in the case of HAPPY at AC. Further, we found that the factor scores of trustworthiness correlated weakly with the subjects' selections of "cooperate" $(r=$ $0.4715)$. Moreover, the average value of the three factors correlated weakly with the subjects' selections $(r=0.3967)$.

We verified the relationship between facial expressions and factor scores and found that HAPPY induced a higher friendliness impression, but ANGRY induced a lower one than any other expressions. A comparison of the factor score of friendliness in the AC between HAPPY and COOL and between ANGRY and COOL revealed that these differences are statistically significant at the .05 level ( $p$-value, $t$-test). Moreover, we found that ANGRY induce a higher dominance impression but BOW and AVERT induce a lower dominance impression than any other expressions. The comparison of the factor score of dominance in the $A C$ between these expressions and $\mathrm{COOL}$, are statistically significant at the .05 level.

\section{Discussion}

The results revealed that facial expressions and history have a significant effect on the impressions related to friendliness, trustworthiness, and dominance. We also discovered that the impressions influenced the subjects' decisions to select "cooperate" or "defect." Further, the results revealed that in the negotiation game, humans behave partly like that suggested in our model. This model can be used to plan the agent's behaviors to make a user cooperate during negotiation or to encourage factors such as friendliness and trustworthiness. It is important to identify the parameters that will be efficient and significant in helping humans design agents and robots.

In this experiment, we used a simple negotiation game. In the future, we will use price negotiation that requires users to exchange messages and facial expressions with each other [18]. Although we used only one history in our experiment, price negotiation will require more histories and also the incorporation of the manner in which the previously proposed moves of a player influenced the other player. Furthermore, according to Thompson et al. [15], not only positive emotional expression but also the expression of extreme irrational behavior can be highly effective in negotiation. Our next aim is to develop a sophisticated model that considers both rational parameters like the evaluation value in game theory and irrational (in fact, rational) behavior like emotional expression in negotiation. Moreover, we will improve the model gradually, observing human behavior by a negotiation game.

\section{Conclusion}

In this paper, we proposed a new decision relevance model for the facial expressions of an animated agent 
based on soft game theory and applied it to a negotiation game. This model can be used to design the agent's and robot's behaviors. In the future, in order to improve our model, we will attempt to investigate other types of games.

\section{Acknowledgments}

This research was partially supported by the Ministry of Education, Culture, Sports, Science and Technology (Grant-in-Aid for Scientific Research (B) 16300032, 2006), by the Research Center for Advanced Technologies, Tokyo Denki University, and by Research Institute for Science and Technology of Tokyo Denki University Q06J-14/ Japan.

\section{References}

[1] Baylor, A.L., Rosenberg-Kima, R.B and Plant, E.A. Interface agents as social models: The impact of appearance on females' attitude toward engineering. In Proc. CHI '06, (2006), 526-531.

[2] Eckel, C. and Wilson, R. Why fairness? Facial expressions, evolutionary psychology and the emergence of fairness in simple bargaining games. Workshop on the Workshop II conference, (1999).

[3] Fukayama, A., Ohno, T., Mukawa, N., Sawaki, M. and Hagita, N. Messages embedded in gaze of interface agents - impression management with agent's gaze. In Proc CHI2002, (2002), 41-49.

[4] Goetz, J., Kiesler, S. and Powers, A. Matching robot appearance and behavior to tasks to improve humanrobot cooperation. In Proc. ROMAN 2003, (2003), 5560.

[5] Howard, N. 'Soft' Game Theory. Information and Decision Technologies 16, 3 (1990), 215-227.

[6] Inohara, T. and Nakano, T. Properties of 'soft' games with mutual exchange of inducement tactics.
Information and Systems Engineering 1, 2 (1995), 131148.

[7] Kiesler, S., Sproull, L. and Waters, K. A Prisoner's Dilemma experiment on cooperation with people and human-like computers. Journal of Personality and Social Psychology 70, 1 (1996), 47-65.

[8] Koda, T. and Maes, P. Agents with faces: The effect of personification. In Proc. Roman '96, (1996).

[9] Ortony, A., Clore, G.L. and Collins, A. The Cognitive Structure of Emotions. Cambridge Univ. Press, 1998.

[10] Parise, S., Kiesler, S., Sproull, L. and Waters, K. My partner is a real dog: Cooperation with social agents. In Proc. CSCW '96, (1996), 399-408.

[11] Picard, R.W. Affective Computing. The MIT Press, 1995.

[12] Poggi, I., Pelachaud, C. and Caldognetto, E.M. Gestural mind markers in ECAs. In Proc AAMAS 2003, (2003), 1098-1099.

[13] Prendinger, H. and Ishizuka, M. Life-Like Characters: Tools, Affective Functions, and Applications. Springer, 2003.

[14] Rist, T., Andre, E. and Baldes, S. A flexible platform for building applications with life-like characters. In Proc Intelligent User Interfaces 2003, (2003), 158-165.

[15] Thompson, L.L., Medvec, V.H., Seiden, V. and Kopelman, S. Poker face, smiley face, and rant' $n$ ' rave: Myths and realities about emotion in negotiation. Blackwell Handbook in Social Psychology 3 (2000).

[16] Velasquez, J. Modeling emotions and other motivations in synthetic agents. In Proc. AAAI 97, (1997), 10-15.

[17] Yuasa, M. Strategies using facial expressions and gaze behaviors for animated agents. In Proc. AMIRE 2005, (2005), 337-343.

[18] Yuasa, M., Yasumura, Y. and Nitta, K. Negotiation support tool using emotional factors. In Proc. IFSANAFIPS2001, (2001), 2096-2911. 